\title{
Dispersion Properties of Up-doping Photonic Crystal Fibers
}

\author{
Huda M. Mohammad Nawfal Y. Jamil \\ Department of Physics \\ College of Science \\ University of Mosul
}

Email: huda_masood2@yahoo.com nawfaljamil@yahoo.com

\author{
Abdulghafoor I. Abdullah \\ Department of Physics \\ College of Education \\ University of Mosul
}

ghaa4@yahoo.com

(Received 12/5/2013 ; Accepted 16/7/2013)

\begin{abstract}
In this papers the novel photonic crystal fibers (PCFs) which consist of air holes surrounded by other substances with triangular lattice arrangement have been assumed. A Full-vector plane wave expansion method (FV-PWEM) has been implemented to calculate the dispersion properties of PCF which possess circular air holes with hole radius $\boldsymbol{R}=\mathbf{0 . 2 5} \boldsymbol{\mu m}$, lattice constant $\boldsymbol{\Lambda}=\mathbf{1} \boldsymbol{\mu m}$, and air filling fraction $\boldsymbol{f}=\mathbf{0 . 2 3}$. Then the effect of up-doping has been studied using two different germanium dioxide percentages. As a result, a tuning of the total dispersion curve has been obtained.
\end{abstract}

Keywords: Doping, photonic crystal, photonic crystal fibers.

\section{خصائص التثتت للألياف البلورية الفوتونية ذات التطعيم العالي}

\section{الملخص}

في هذا البحث، فُرض نوع جديد من الأليف البلورية الفوتونية (PCFs) التي تتك ـون مه ـن فج ـوات

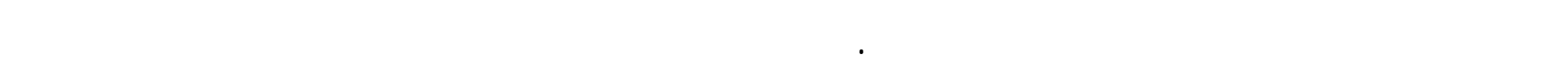

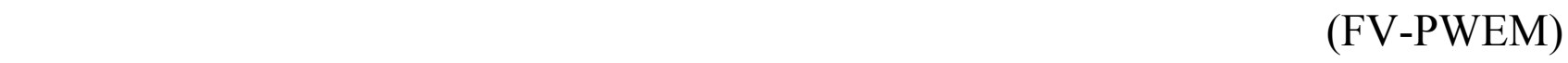
قطرها

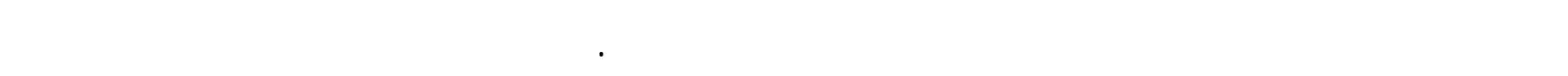
لمنحف التشت الكلي.

\section{INTRODUCTION}

Photonic crystal fibers (PCFs) or Photonic bandgap (PBG) fibers are fibers with an internal periodic structure made of capillaries, filled with air, laid to form a geometrical lattice (triangular, square, honeycomb, ... etc.). These fibers are a novel class of optical fibers, and can be considered as the state-of-the-art fibers. Combining properties of optical fibers and photonic crystals possess a series of unusual properties which are impossible to achieve in classical fibers (Buczynski, 2004) such as endlessly single mode, ultra flattened dispersion, zero dispersion wavelength (ZDW) at a visible region, and a very high negative dispersion, all of these properties can be controlled by manipulating the parameters of the 
PCF such as lattice pitch $\Lambda$ (lattice constant), air hole shape, air hole diameter $d$, air filling fraction $f$, and type of lattice. (Fig. 1) shows the proposed PCF with triangular lattice of air holes with two rings of holes.

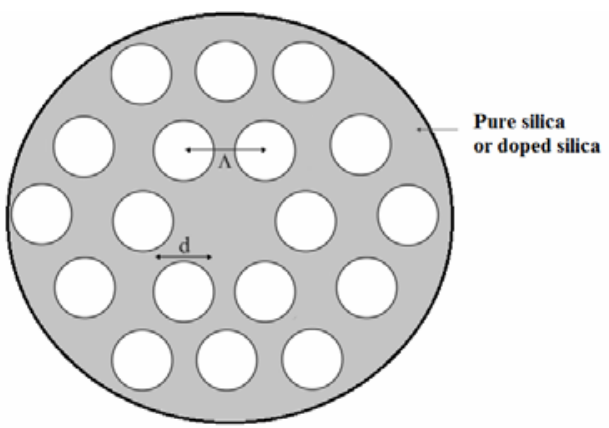

Fig. 1: PCF with triangular lattice of air holes.

PCFs are usually manufactured from pure silica into which a periodic array of holes is formed and runs axially along the entire fiber length. No dopants are used, as it is the arrangement of the air spaces that determines mode confinement (Buck, 2004).

In conventional germanium-depend fibers, the mirror is due to a total internal reflection (TIR) at the core-cladding interface, and at the modal calculation essentially involves satisfies the phase condition. In contrast with PBGFs, where the mirrors are associated with Bragg reflection, a modal calculation needs to be preceded by a separate band structure calculation of the cladding (White et al., 2001). Therefore different methods for PCFs have been developed and discussed such as the Finite element method (FEM) (Najafi et al., 2010) and the Plane wave expansion method (PWEM) (Salcedo-Reyes, 2012) to calculate this band structure. The most common of these methods is the full-vector plane wave expansion method (FV-PWEM), in which the field is written as a superposition of plane wave. FV-PWEM is accurate and robust, and has been found to be a simple and efficient to obtain the dispersion relation of different PBG configurations.

In this paper, different germanium dioxide percentages and accordingly different silica percentages $\left(4.1 \% \mathrm{GeO}_{2}+95.9 \% \mathrm{SiO}_{2}\right.$, and $\left.7 \% \mathrm{GeO}_{2}+93 \% \mathrm{SiO}_{2}\right)$ doped silica PCF have been studied, which are known as up-doped PCFs, because they possess a high refractive index compared with the refractive index of pure silica. The dispersion properties have been calculated with FV-PWEM, and the results of different up-doping have been compared with pure silica PCFs $\left(100 \% \mathrm{SiO}_{2}\right)$ which have been measured in the same way.

\section{THEORETICAL DETAILS}

\section{Full-Vector Plane Wave Expansion Method:}

FV-PWEM is a computational technique to solve the Maxwell's equations by formulating an eigenvalue problem. This method is useful in calculating modal solutions over an inhomogeneous or periodic geometry. The z-direction in PCF taken to be uniform, and the transverse field component obeys the following vector wave equation (Zhu and Brown, 2001): 


$$
\begin{aligned}
& \left(\nabla_{t}^{2}+k_{o}^{2} \varepsilon\right) E_{t}+\nabla_{t}\left(E_{t} \cdot \nabla_{t} \ln \varepsilon\right)=\beta^{2} E_{t} \\
& \left(\nabla_{t}^{2}+k_{o}^{2} \varepsilon\right) H_{t}+\nabla_{t} \ln \varepsilon \times\left(\nabla_{t} \times H_{t}\right)=\beta^{2} H_{t}
\end{aligned}
$$

where $\boldsymbol{\beta}$ is the propagation constant of the mode, $\boldsymbol{H}_{t}$ and $\boldsymbol{E}_{t}$ are the transverse components of the magnetic and electric field, respectively. $k$ is the wavevector, and $\varepsilon=\varepsilon(x, y)$ is the transverse dielectric constant profile, which is considered as a periodic function of the space and can be expanded using Fourier series (Zhu and Brown, 2001) :

$$
\varepsilon(r)=\sum_{G} \varepsilon_{G} e^{i \vec{G} \cdot \vec{r}}, \ln \varepsilon(r)=\sum_{G} K_{G} e^{i \vec{G} \cdot \vec{r}}
$$

Where $\vec{G}$ is the grid vector in the reciprocal space.

The transverse field $\boldsymbol{E}_{t}$ can also be expanded using Fourier series (Zhu and Brown, 2001):

$$
E_{t}=\sum_{t}\left\{E(G, x) e^{i \vec{G} \cdot \vec{r}} \hat{x}+E(G, y) e^{i \vec{G} \cdot \vec{r}} \hat{y}\right\}
$$

Expanding eigen-function and inversed permittivity functions to the Fourier series by the reciprocal lattice vectors and substituting them in master equation $\boldsymbol{\nabla} \times \frac{1}{\varepsilon(r)} \boldsymbol{\nabla} \times \boldsymbol{E}(\boldsymbol{r})=\left(\frac{\omega}{c}\right)^{2} \mathbf{E}(\mathbf{r})$ will give us the next equation (Zhu and Brown, 2001):

$$
\begin{aligned}
-|G|^{2}\left(E_{G x} \hat{x}\right. & \left.+E_{G y} \hat{y}\right)+k_{o}^{2} \sum_{G} \varepsilon_{G-G}\left(E_{G x} \hat{x}+E_{G y} \hat{y}\right) \\
& -\sum_{\vec{G}} k_{\vec{G}-\vec{G}}\left[\left(G_{x}-\dot{G}_{x}\right) E_{G_{y}}\right]\left(G_{x} \hat{x}+G_{y} \hat{y}\right) \\
& =\beta^{2}\left(E_{G x} \hat{x}+E_{G y} \hat{y}\right)
\end{aligned}
$$

Solving this system numerically we obtain the number of eigen-frequencies corresponding to some value of $\boldsymbol{k}$ in an irreducible Brillouin zone (IBZ), which is the smallest section of the full Brillouin zone that is bounded by symmetry lines of the Bravaies lattice to which the Brillouin zone is attached.

\section{Dispersion Consideration}

An optical fiber is a dispersive medium, i.e., different frequencies travel at different speed inside the fiber. This is attributed to the frequency-dependence of the refractive index of silica which is commonly referred to as material dispersion $\left(\boldsymbol{D}_{M}\right)$. Difference in refractive index between the core and cladding induces another type of dispersion, commonly referred to as waveguide dispersion $\left(D_{W}\right)$. The total dispersion of the fiber is the sum of these two types of dispersion (Wong, 2003). 


$$
D(p s / n m / k m)=D_{M}+D_{W}
$$

Effects of dispersion are accounted for by expanding the propagation constant $\boldsymbol{\beta}$ around the carrier frequency $\omega_{0}$ of the pulse traveling through the fiber (Lehtonen, 2002). In the wave propagation simulations only the fundamental mode will be treated, therefore we concentrate on $\boldsymbol{\beta}$. Instead of plotting $\omega$ as a function of $\boldsymbol{k}_{z}=\boldsymbol{\beta}$ as in normally done for band structure, here $n_{\text {eff }}$ is plotted as a function of the free space wavelength $\lambda=2 \pi c / \omega$ (where $\mathbf{c}$ is the velocity of light in a vacuum). The propagation constant takes the band structure of the fiber into account giving $n_{\text {eff,bandstructure }}=c \beta / \omega$. The frequency dependence of the effective refractive index arising from the silica is subsequently included as (Hilligsoe, 2003):

$$
\boldsymbol{n}_{\text {eff }}=\boldsymbol{n}_{\text {material }}+\boldsymbol{n}_{\text {eff }, \text { bandstructure }}-\boldsymbol{n}_{\text {constat }}
$$

where refractive index of silica $\boldsymbol{n}_{\text {constat }}=1.45$. and $\boldsymbol{n}_{\text {material }}$ has been calculated from the Sellmeier formula (Hilligsoe, 2003):

$$
n_{\text {material }}{ }^{2}(\lambda)=1+\sum_{j=1} \frac{B_{j}^{2}}{1-\left(\frac{\lambda}{\lambda}\right)^{2}}
$$

Here $\lambda$ is the wavelength of incident light, $\lambda_{j}$ is an atomic resonance wavelength, and $\boldsymbol{B}_{j}$ is the oscillator strength of transitions. For the calculations of three PCFs under investigation the parameters in Table (1) have been used (Adams, 1981):

Table 1 : Sellmeier Constants for pure silica and two up-doped glass

\begin{tabular}{|c|c|c|c|c|c|c|}
\hline & $\xi_{1}$ & $\bar{S}_{\Sigma}$ & $\bar{\Xi}_{\Sigma}$ & $A_{1}$ & $A_{2}$ & $A_{3}$ \\
\hline $\begin{array}{c}\text { Pure silica } \\
100 \% \mathrm{SiO}_{2}\end{array}$ & 0.6961663 & 0.4079426 & 0.8974974 & 0.068043 & 0.1162414 & 9.896161 \\
\hline $\begin{array}{c}4.1 \% \mathrm{GeO}_{2} \\
+\end{array}$ & 0.686717749 & 0.43481505 & 0.89656582 & 0.07267518 & 0.11514351 & 10.002398 \\
\hline $\begin{array}{c}95.9 \% \mathrm{SiO}_{2} \\
7 \% \mathrm{GeO}_{2}+ \\
93 \% \mathrm{SiO}_{2}\end{array}$ & 0.6869829 & 0.44479505 & 0.79073512 & 0.07807858 & 0.11551840 & 10.436628 \\
\hline
\end{tabular}

The total dispersion (chromatic dispersion) of a PCF is directly calculated from the effective index of the fundamental mode $n_{\text {eff }}$ versus the wavelength using (Saitoh and Koshiba, 2005) :

$$
D(p s / n m / k m)=-\frac{\lambda d^{2} n_{e f f}}{c}
$$


In real terms the total dispersion is a measure of the change in the refractive index $n_{\text {eff }}$ as function wavelength, for this reason dispersion measurement can be read as going positive or negative.

\section{CALCULATIONS AND RESULTS}

PCFs with claddings of triangular lattice of circular air holes, with lattice pitch $\Lambda=1 \mathrm{~mm}$, air hole radius $R=d / 2=0.25 \mathrm{~km}$ and air filling fraction $f=\frac{2 \pi R^{2}}{\sqrt{3} A^{2}} \approx 0.23$, with two rings of holes as shown in (Fig. 1) have been considered.

In order to investigate the up-doping effect on fiber dispersion properties, two updoped glass PCFs have been used beside the pure one (100\% silica PCFs), then the material refractive index ( $\left.\boldsymbol{n}_{\text {material }}\right)$ of these designs has been calculated as a function of wavelength as shown in (Fig. 2) using Sellmeier formula (Eq. 8), when (Fig. 3) shows the band structure refractive index ( $\boldsymbol{n}_{\text {eff,bandstructure }}$ ) which has been calculated by solving the eigen-value equation (Eq. 5). For two cases, a difference between the refractive index for different up-doped percentages is observed, and the refractive index decreases with increasing wavelength for all PCFs. In addition, it is worthy to note that the effective index of up-doped PCFs increases above that for pure silica PCF (so-called up-doping) for two cases.

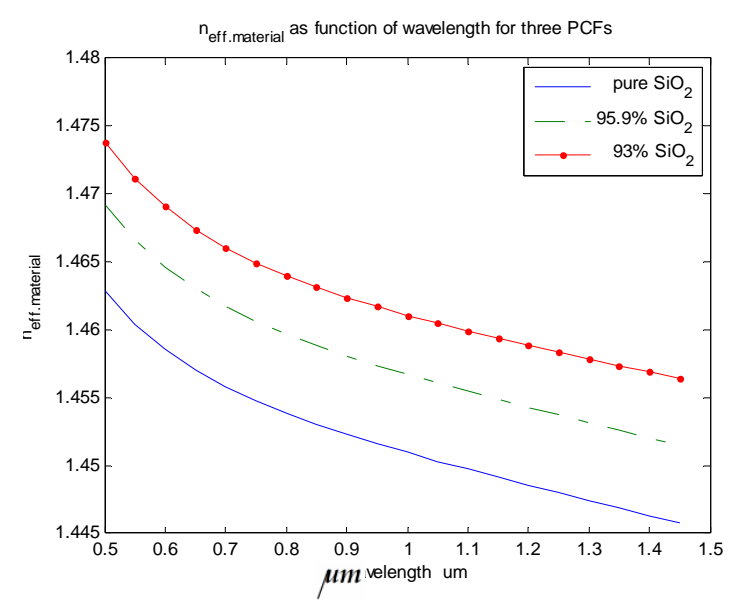

Fig. 2: Material refractive index $\left(n_{\text {material }}\right)$ as a function of wavelength, for pure silica and two different up-doped glass PCFs

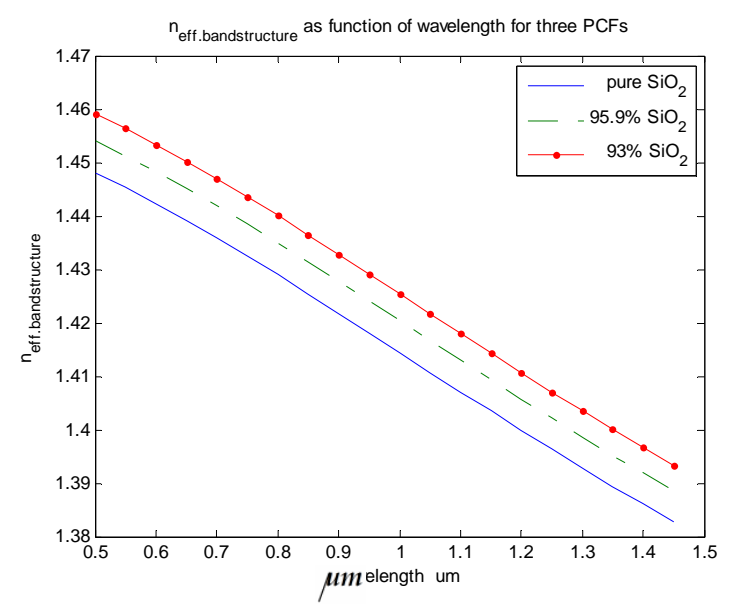

Fig. 3: Band structure refractive index (neffondstructure) as a function of wavelength for pure silica PCF, and two different up-doped glass PCFs

At last, the total (chromatic) dispersion of light in all PCFs has been calculated using Eq. 9, and the results shown in (Fig. 4) as a function of wavelength. It can be clearly seen that the dispersion curves do not have a gradual change between pure PCF and the other two up-doped PCFs, which result from the fact that the total dispersion $(D)$ is the consequence of compensation of material dispersion $\left(D_{M}\right)$ and waveguide dispersion $\left(D_{W}\right)$, or sum of these two types of dispersion, as mentioned in Eq. 6, and because the up-doped effects largely appear on each of the material refractive index and band structure refractive index 
with different variations, as shown in previous figures, then the total dispersion does not have a gradual variation with gradual doped.

As clearly shown in (Fig. 4), there is a high negative dispersion $(118.3 \mathrm{ps} / \mathrm{nm} / \mathrm{km})$ at $1.3 \mu \mathrm{m}$, as well as nearly flattened in $0.82-0.96 \mu \mathrm{m}$ range with a small negative dispersion such as $\approx-13.5 \mathrm{ps} / \mathrm{nm} / \mathrm{km}$ at $0.9 \mu \mathrm{m}$ for silica up-doped PCFs with $93 \% \mathrm{SiO}_{2}$, as shown in the enlarged graphic of the total dispersion in (Fig. 5).

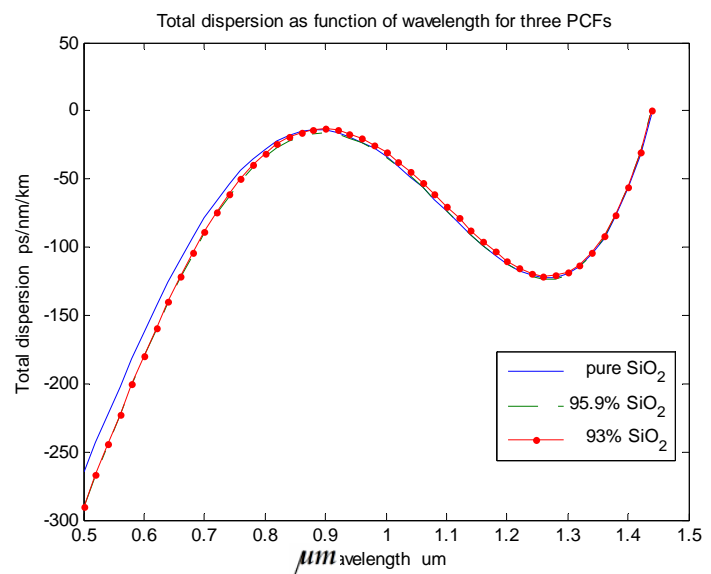

Fig. 4: Total dispersion of light as a function of wavelength, for pure silica PCF, and two different updoped glass PCFs.

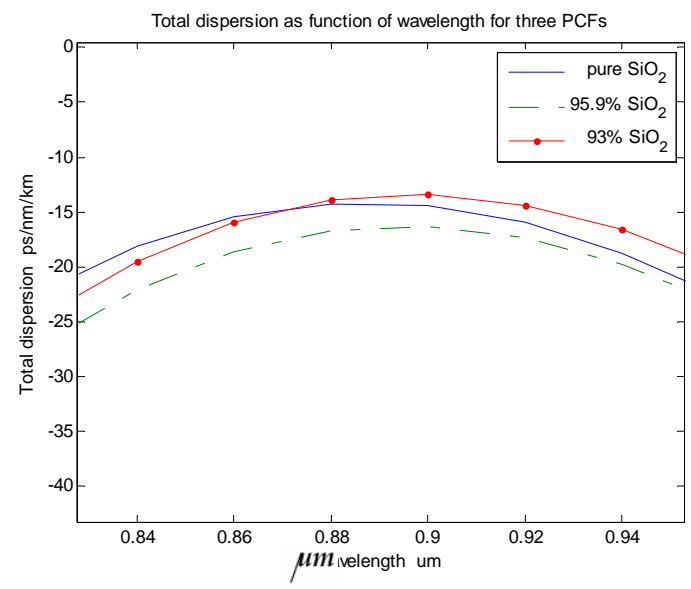

Fig. 5: Enlarged graphic of the total
dispersion at o.8
telecommunication window for
pure silica PCF, and two
different up-doped glass PCFs.

\section{DISCUSSION}

The results of effective refractive index illustration are obtained using FV-PWEM. This work shows the up-doped effects on each of the material refractive index, band structure refractive index, effective refractive index, and as a result total dispersion of proposed PCF. which can be accounted as a result of changing the refractive index contrast $(\delta)$ which offers a general idea of the scattering strength and defined as the ratio between the refractive index of the high dielectric constant material $\left(\boldsymbol{n}_{h}\right)$ and the low dielectric constant material $\left(\boldsymbol{n}_{l}\right), \delta=\boldsymbol{n}_{h} / \boldsymbol{n}_{\boldsymbol{l}}$.

It can also be concluded that the total dispersion is shown to be dominated by both material dispersion $\left(D_{M}\right)$ and waveguide dispersion $\left(D_{W}\right)$ in this type of geometry PCFs (Triangular PCFs) even though the difference in effect is not similar, leading to a compensation of the total dispersion in little variation, in contrast with the conventional fiber.

The proposed PCFs which have a high negative dispersion as well as a flattened dispersion at a transmission wavelength bands, $1.3 \mu \mathrm{m}$ and $0.82-0.96 \mu \mathrm{m}$ respectively, produce a wide range of applications and can be used for various purposes in the field of optical telecommunication, where the general advantage of negative dispersion is being 
important in ultra-short-pulse generation, which is a very active field of research, since it has found applications in high speed communications.

\section{CONCLUSION}

The up-doping fibers can tune and modulate the dispersion, therefore the up-doping in triangular PCFs provides an additional manner to change the total dispersion, which could be an intersecting tendency in PCFs design for dispersion engineering. As well as possessing a negative dispersion which makes the up-doping triangular PCFs as a helpful structure which can be used to transverse the light with relatively low losses and ultra-shortpulse.

\section{REFERENCES}

Adams, M.J. (1981). "An Introduction to Optical Wave-guides". Chichester: John Wiley and Sons, Ltd.

Buck, J.A. (2004). "Fundamental of Optical Fibers". 2nd ed., John Wiley and Sons, Hoboken New Jersey.

Buczynski, R. (2004). "Photonic Crystal Fibers". Physica Polonica, 106, 2.

Hilligsoe, K.M. (2003). Wave propagation in photonic crystal fibers. University of Arahus and NKT Academy, Department of Physics and Astronomy and Department of Chemistry, Denmark, M.Sc. Thesis.

Lehtonen, M. (2002). Supercontinuum generation in photonic crystal fiber. Helsinki University of Technology, Department of Electrical Engineering, Finland, M.Sc. Thesis.

Najafi, A.; Jalalkamali, M.; Moghadamzadeh, S.; Bolorizadeh, M.A. (2010). "Finite element method analysis of photonic crystal fiber band structure". Photonics and Optoelectronic, 1-4.

Saitoh, K.; Koshiba, M. (2005). Numerical modeling of photonic crystal fibers. $J$. Lightwave Technology, 23, 11.

Salcedo-Reyes, J.C. (2012). Effective index of face-centered-cubic and hexagonal closepacked 250nm-SiO ${ }_{2}$ based colloidal crystals. J. Nanophotonics, $\mathbf{6}, 1$.

White, T.P.; McPhedran, R.C.; . Botten, L.C.; Smith, G.H.; De, C.; Stereke, M. (2001). Calculation of air-guided modes in photonic crystal fibers using the multipole method. Optics Express, 721(9), 13.

Wong, K.L.G. (2003). Nonlinear optics in photonic crystal fiber. M.Sc. Thesis, University of Auckland, New Zealand.

Zhu, Z.; Brown, T.G. (2001). Analysis of the space filling modes of photonic crystal fibers. Opt. Express 8, 547. 